\title{
LA IDEA DE "VIVIDO-PROYECTADO". UN CRITERIO PARA INTERPRETAR LAS RELACIONES INTERSUBJETIVAS EN EL CAMPO DE LAS CIENCIAS SOCIALES ${ }^{1}$
}

The idea of the "lived-projected". A criteria for interpreting the intersubjective relationships in the field of the social sciences

Rubén Leal Riquelme*

\section{Resumen}

Nos proponemos mostrar que la idea de vivido-proyectado constituye una herramienta útil para continuar el estudio de la teoría de los motivos. La noción vividoproyectado puede ser asumida como un recurso metodológico para interpretar los proyectos de acción $\mathrm{y}$, a la vez, evitar algún tipo de dualismo. Se trata, entonces, de reflexionar acerca de algunos tipos de relaciones que puede establecer la conciencia del actor en los procesos de articulación de las vivencias cuando, especialmente, se pretende desarrollar relaciones sociales con otros actores en la vida cotidiana. A nuestro parecer, la idea de vivido-proyectado y su aplicación puede trascender estudios epistemológicos para incursionar en el campo de la ética y más especialmente en las relaciones que establece un profesor con sus estudiantes, como es el caso que abordaremos en esta ocasión.

Palabras clave: Fenomenología, teoría de la acción, vivido-proyectado, proyectos de acción, relaciones intersubjetivas.

\section{Abstract}

In the present work we propose to show that the idea of "lived-projected" is a useful instrument to continue the study of the theory of motives. In this context, we will reflect about the notion of "lived-projected", for it seems to us that it can be assumed as a methodological resource to interpret the projects of action and, at the same time, to avoid some kind of dualism. Then, our purpose is to reflect about some types of links that the conscience of the actor, in the process of the articulation of lived experiences can establish when, especially, the intention is to develop social relationships with other actors in current life. We believe that the idea of "lived-projected" and its application can go further than the epistemological studies. We think that it is possible to be applied into the field of ethics and especially in the relationships that a teacher establishes with his/her students. Indeed, we focus the analysis in this last case.

Key words: Phenomenology, theory of action, lived-projected, projects of action projects intersubjetive relationships.

\footnotetext{
${ }^{1}$ Artículo derivado del Proyecto Mayor Científico Tecnológico No 3732-10, financiado por la Dirección de Investigación y Postgrado de la Universidad de Tarapacá. Parte de él fue presentado en el III Congreso Iberoamericano de Filosofía de la Ciencia (Buenos Aires, 2010).
} 


\section{LA IDEA DE VIVIDO-PROYECTADO. NATURALEZA, SENTIDO Y ALCANCES}

Para proponer la noción de vivido-proyectado hemos considerado, básicamente, las ideas de duree —duración interna - que nos presenta Henri Bergson (1993) y dos planteamientos que formula Alfred Schütz, uno en el ámbito de la teoría de los motivos (1974:49-50; 1993:115-120) y otro cuando discute la idea de presente vivido.

En relación con la idea de duración interna, pareciera que la distinción entre tiempo interno y tiempo externo propiciada por Bergson si bien puede asumirse como el surgimiento de un nuevo tipo de dualismo, propio del modo de pensar moderno que expresaría Hegel (1968) no es menos cierto que para nuestro objetivo, la idea de tiempo interno forma parte de la conciencia del actor que, además, contiene la idea de continuum (1993:483ss.). De manera que en la conciencia es posible integrar y articular las experiencias que el propio Sujeto ha tenido en su vida cotidiana. Por otra parte, esta idea de continuum puede constituir un recurso útil al momento de conocer las vivencias ${ }^{2}$ y las interpretaciones de las acciones, especialmente en cuanto al futuro de los proyectos de acción.

En el caso de Schütz, cuando propone la teoría de los motivos — a partir de lo propuesto por Weber - procede a establecer una distinción entre motivos porque y motivos para, sosteniendo que en el segundo tipo las orientaciones pueden ser observadas en la ejecución de los actos e, incluso, en los propios proyectos. En este punto, Schütz procede a tomar de Weber la idea de motivo, idea que, a la vez, procede a criticar. Pero detengámonos en lo que propone Weber y que cuestiona Schütz.

Para Max Weber, el motivo es una especie de configuración o de contexto de significado que tiene presente un actor y/o un observador, y su propósito es asumir ese contexto como fundamento significativo de una conducta (Leal, 2009:135-151). Es en este momento cuando surgen los cuestionamientos que realiza Schütz y que a nuestro parecer se sustentan en tres consideraciones principales:

1. La noción de motivo formulada por Weber integra a dos cuestiones que se muestran diferentes. Por una parte, nos referimos al contexto de significado que el actor siente - subjetivamente - y, por otra, se alude al contexto de significado que el observador supone que corresponde al fundamento de la conducta ejecutada. Para Schütz, esto es inadmisible, ya que desde la perspectiva de la teoría del significado aquello que el actor siente, subjetivamente, y aquello que supone el observador corresponden a dos consideraciones diferentes, las que, a su vez, son inconmensurables. 2. Por su parte, para Weber, la conducta es una especie de datum, discreto y unificado, gracias al cual es posible operar de modo inmediato; es decir, la acción

\footnotetext{
${ }^{2}$ Asumimos la idea de vivencia como un fenómeno experimentado por un actor social en diversas circunstancias y que ha representado en su conciencia. Las representaciones de las experiencias conforman las vivencias y ellas se encuentran en estado presente en la conciencia. Esto último es posible gracias a que en la conciencia no existe pasado, presente, ni tiempo futuro. En Bergson, el tiempo interno no es cuantificable y gracias a ello se puede afirmar que el actor mantiene vivencias en estado presente, a las cuales recurre al momento de formular proyectos de acción, por ejemplo.
} 
puede ser realizada sin necesidad de algún tipo de investigación vinculada al principio de unidad que la propia conducta del actor tiene, por ejemplo. Sin embargo, Schütz estableció que la unidad de la acción es subjetiva en su propio fundamento, es decir, ella depende del Aquí y del Ahora en el cual se establece el proyecto. De manera que el fundamento significativo de la acción, que es percibido como una unidad por el observador, resulta ser un fenómeno relativo a un particular Aquí y Ahora del actor y que, por lo tanto, es menester realizar una suplementación de la misma.

3. Cuando Weber se refiere a los motivos, no se ocupa de la naturaleza del contexto de significado o, al menos, no apunta a su dependencia en relación a la conducta que ejecuta. A nuestro parecer, para Schütz, esta sería la razón por la cual Weber asimila la comprensión esclarecedora o motivacional a una comprensión de tipo observacional, es decir, Weber no precisa si el significado al cual alude considera una acción que, al menos, sea similar al motivo al cual hace referencia, en tanto que para Schütz, el contexto motivacional corresponde a un contexto de significado para el actor.

Por nuestra parte, consideramos que Weber utiliza indiscriminadamente el término motivo, ya que por este concepto alude a los motivos porque y a los motivos para, lo que también nos parece inadmisible. En este punto optamos por recurrir al propio Schütz para esclarecer y distinguir cada uno de estos dos términos. En el caso de los motivos para, la conciencia del actor nos muestra cómo se origina el acto desde un proyecto determinado $\mathrm{y}$, a la vez, nos demuestra cómo esos motivos permiten distinguir a la conciencia del propio acto que realiza un Sujeto; es decir, en el ámbito de los motivos, la conciencia procede a acotar y a esclarecer el contexto y los alcances que son propios del proyecto de acción. A nuestro parecer, el proyecto, de algún modo, se encuentra determinado por el motivo porque, que lo provoca. Así, los motivos serían parte de sistemas subjetivos en el ámbito de la planificación que realiza un actor y desde aquí emergen las aspiraciones futuras, sean ellas laborales, sean actividades cotidianas o relacionadas con el uso del tiempo de descanso, por enunciar solo tres ejemplos.

Lo anterior, permite afirmar que el para tiene un sentido futuro en el caso del motivo, es decir, cuando se ejecuta una acción nos encontramos con un proyecto específico que orienta al acto. De manera que el proyecto y el propio acto son orientados por un acto de fantaseo, asumiendo a este último como un ejercicio $a$ priori que compromete el tiempo futuro perfecto. Cuando el actor fantasea opera como si se hubiera realizado el proyecto, de lo cual podríamos inferir que la unidad de la acción se constituye gracias a ese proyecto y sus alcances dependerán, entonces, del modo como el actor procede a formularlo. De aquí, también, se puede afirmar que el futuro en el cual se encuentra el mundo de los sucesores, por ejemplo, se percibe completamente indeterminado; más aún, todo parece indicar que ese mundo es indeterminable. Al respecto, Schütz nos señala

(...) Si el mundo de los predecesores es algo fijado y determinado por completo, si el mundo de los consociados es libre y el de los contemporáneos es 
probable, el de los sucesores es por completo indeterminado e indeterminable. Nuestra orientación hacia nuestros sucesores no puede llegar más que a esto: que vamos a tener algunos. Ninguna llave puede abrir la puerta de este reino. En efecto, este último método se basa en nuestra experiencia de los predecesores, los consociados y los contemporáneos, y no hay ningún principio que nos permita extenderlo al mundo de los sucesores (1993:242).

No obstante lo expresado por Schütz, pensemos en la posibilidad de algunas aproximaciones al mundo de los sucesores, claro, teniendo presente que ese ejercicio sólo se puede realizar de una manera indirecta $o$, al menos que aceptemos que nuestra aproximación se mantiene en el ámbito del fantasear. Si aceptamos que los sucesores son actores similares a mí y que ellos vivirán en un mundo cotidiano que ha sido construido por mis antecesores y sobre el cual durante mi vida también he tenido la posibilidad de intervenir para conformar la realidad cotidiana que hoy observamos, esa realidad que ayudamos a construir, entonces, será el ámbito en el cual nacerán mis nietos, es decir, corresponderá a la realidad que ellos encontrarán cuando yo no esté. Por su parte, aceptando que mis nietos modificarán el mundo que reciben, ese mundo influirá de manera sustancial en las experiencias y en los proyectos que formulen mis descendientes, sea para conformar sus vidas personales, sea para construir las relaciones sociales que compartirán en la vida cotidiana. En este sentido, parece posible tener alguna incidencia en la vida de mis predecesores. En el mismo sentido, aceptamos que es posible aproximarnos, al menos teóricamente, a los proyectos de mis nietos: hoy planto algunos árboles frutales en el patio de mi casa, fantaseo que mis predecesores, directos en este caso, podrán gustar de sus frutos, especulo que esos árboles les proporcionarán sombra durante los meses de verano, fantaseo que transcurridos treinta años no producirán frutos o servirán de combustible para la chimenea. En cada una de las situaciones aludidas, de algún modo, influyo en los proyectos de acción y en las conductas de quienes aún no han nacido. Claro, también pudiera suceder que yo no tenga descendientes y en ese caso sólo he especulado acerca de lo que sucederá.

Aceptando que nuestro ejemplo se refiere a una situación particular, no podemos desconocer que en él se observa una lógica que el propio Schütz respalda cuando expresa que

(...) como también lo señaló Husserl con la mayor claridad, nuestras protecciones y anticipaciones de lo venidero son esencialmente referencias vacías a los horizontes abiertos, que los sucesos futuros pueden llenar o "hacer estallar" (...). En otras palabras, toda experiencia lleva consigo su propio horizonte de indeterminación (tal vez una indeterminación en cierta medida determinable) que se refiere a experiencias futuras (1974:262).

Desde otro ángulo y en relación a los motivos para, hemos tenido a la vista, una segunda consideración para referirnos a la idea de vivido-proyectado, tal es la 
idea de "protención", noción que toma de Husserl (262). Conforme a ella, Schütz sostiene que todo suceso contiene aspectos relacionados con el pasado y con el futuro mensurable; es decir, mediante la idea de "protención" reconoce que las experiencias, las vivencias, los proyectos de acción y los actos, contienen aspectos que el actor ha vivido en el pasado; más aún, los actos futuros que él realizará también contienen (...) "anticipaciones o previsiones de sucesos más distantes en el tiempo, con los cuales, según prevé, estará relacionada la experiencia presente" (262). Es decir, mediante las protenciones el actor establece una especie de síntesis entre aquello que ha vivido y lo que se propone vivir, para lo cual sus acciones siguen una lógica que se sustenta en las "estructuras típicas" que le han sido útiles en situaciones ya vividas, de manera que no existiría razón suficiente para cambiarlas. Aprovechemos de señalar que las "estructuras típicas" y las vivencias constituyen los insumos que conforman el acervo de conocimiento. ${ }^{3}$

Lo expresado, nos permite decir que en la noción de "vivido-proyectado" subyacen las ideas de "duración interna" y de "motivos para". A su vez, esta noción puede ser usada en los procesos interpretativos y en la propia formulación de los proyectos de acción. Del mismo modo, consideramos que esta idea puede transformarse en un recurso que facilita el estudio de las relaciones que construyen dos actores, por ejemplo. En este contexto, Schütz, desde la noción de mundo de la vida, alude a los diversos tipos de relaciones sociales que establecen los actores y que nosotros aplicaremos más adelante en las relaciones entre maestro y discípulo

(...) "Mi mundo social, con los alteregos que contiene, está ordenado, conmigo como centro, en asociados, contemporáneos, predecesores y sucesores (...), mediante lo cual yo y mis diferentes actitudes hacia Otros instituyen estas múltiples relaciones. Todo esto se da en diversos grados de intimidad y anonimia" ${ }^{4}$ Por su parte, el mundo de los contemporáneos:

\footnotetext{
${ }^{3}$ En el pensamiento de Schütz corresponde a una especie de almacenamiento de vivencias, ellas incluyen a las experiencias directas que ha tenido el actor y también aquellas que le han sido transmitidas por otro actor, sea de manera oral o escrita. El conocimiento que constituye el "acervo" proviene — entonces - de las experiencias y vivencias del Sujeto y de los conocimientos que ha heredado socialmente. De manera que el "acervo" sirve de "insumo" y ayuda al actor en su desempeño en la "vida cotidiana y, por otra parte, le permiten asumir, describir y resolver, nuevas situaciones que experimenta cotidianamente. En el caso del observador-científico, el "acervo de conocimiento" también desempeña esa función de "insumo"; sin embargo, los recursos que obtiene gracias al "acervo" les son útiles al momento de realizar procesos interpretativos. Cfr., Leal (2006:208-210).

${ }^{4}$ Schütz, A. (1995:138). La idea de asociados que usa este autor corresponde al Otro/s con quien/es el actor se relaciona "cara a cara" en la "vida cotidiana". Esta relación adopta un carácter subjetivo, pues se trata de la conciencia del Otro/s que permite el surgimiento del fenómeno intersubjetivo (social). Los contemporáneos son los sujetos con quienes tengo relaciones intersubjetivas y donde ellas no necesariamente tienen un carácter directo, pues también sucede, por ejemplo, que tenemos relaciones indirectas con un amigo que no vemos desde la época de estudiante; sin embargo, transcurridos los años volvemos a verlo o, simplemente, iniciamos una relación postal. Según Schütz, nuestro condiscípulo ahora no es el mismo desde la última vez que compartimos, ya que tanto él como Yo hemos cambiado. Más aún, podríamos pensar que no volveremos tener una relación directa y, de ocurrir esto último, ya es otra persona
} 


\section{Rubén Leal}

(...)" es el mundo inmediato, dentro del cual es posible la experiencia directa y relativamente íntima de Otros. El Mitwel es un mundo de experiencia mediata, pero contemporánea, dentro del cual puede obtenerse una experiencia indirecta y relativamente anónima de Otros. El Vorwelt alude a experiencias del pasado histórico. El Folgewelt se refiere al futuro, del cual ninguna experiencia es posible, pero hacia el cual puede existir una orientación" (138).

Es verdad que con mis sucesores no puedo establecer una experiencia directa ya que aún no han nacido y no conviven conmigo; sin embargo, también existe la posibilidad de que mis acciones y mis propias conductas puedan tener una orientación hacia el mundo de ellos (sucesores). Es en este sentido que podemos utilizar la noción de vivido-proyectado.

Pero hay más, las relaciones intersubjetivas que puedo establecer con un congénere que, entre otras posibilidades, nos llevan a consensuar proyectos para ejecutar en conjunto con ellos en la vida cotidiana; potencialmente, esos proyectos pueden estar orientados a establecer relaciones con nuestros sucesores. En este mismo sentido, si fuera posible enunciarlo de un modo indirecto, podré y podremos relacionarnos socialmente. Claro, no tenemos certeza si este tipo de relaciones se pueden asumir como un tipo especial de relaciones intersubjetivas, de lo que sí estamos ciertos, es que nuestros proyectos tendrán algún efecto en mi nieto, que aún no ha nacido. El efecto puede estar referido a una actitud de aceptación, total o parcial del mismo, o su rechazo también podrá ser parcial o total. De lo que no cabe duda es que mi nieto cambiará el proyecto, al menos para reorientarlo. El tipo de casa, los principios morales que subyacen en la vida de mi familia, la organización social que ayudé a construir en el ámbito de la convivencia, podrán ser aceptados o rechazados, parcial o totalmente por mi nieto. Lo que nos parece nítido, es que mi congénere no podrá abstraerse de esa realidad. No obstante, tengo un alto grado de seguridad que aquello que será motivo de su herencia también será modificado en el transcurso de su vida. Lo expresado, nos permite pensar que la idea de vivido-proyectado no sólo se encuentra en el contexto de las investigaciones acerca de las relaciones intersubjetivas, sino además, ella también nos puede ser útil en la elaboración de una estrategia de trabajo, en el ámbito de la epistemología de las ciencias sociales.

La formulación de un proyecto de acción, por su parte, coloca al actor ante opciones entre las cuales se verá impelido a elegir una de ellas en el ejercicio de sus posibles relaciones con otros actores. Con este solo enunciado es posible imaginar los

con quien me relaciono (Leal, 2001:224-225). Los predecesores son aquellos actores sociales que generacional o temporalmente me anteceden y con los cuales no necesariamente he tenido relaciones intersubjetivas, de manera directa. No obstante, el conocimiento de ellos me ha sido transmitido (indirectamente), y forma parte de mis experiencias y de mi "acervo de conocimiento". Los sucesores corresponden a mis descendientes, a la generación que trasciende mi existencia. Ellos pueden ser descendientes directos o no. Lo que les distingue es que son herederos de mis conocimientos y de toda realización cultural que pude haber realizado durante mi vida. De manera que esa herencia puede ser aceptada, modificada o cambiada totalmente por los sucesores. 


\section{La idea de "vivido-proyectado"}

alcances prácticos que tiene una noción de este tipo. ${ }^{5}$ En general, pensemos en la posibilidad de aceptar al Otro desde un criterio que nos permita tolerar y/o posponer parte de sus aspiraciones, que son diferentes a las que yo me propongo; imaginémonos los alcances prácticos, en la convivencia social y cotidiana que puede tener el conocimiento del Otro, en la elaboración de propósitos comunes. ${ }^{6}$

\section{APLICACIONES DE LA IDEA DE VIVIDO-PROYECTADO}

Si aceptamos que la idea de vivido proyectado, entre otras posibilidades, nos ayuda a potenciar la/s interpretación/es de las acciones que aún no han sido ejecutadas, entonces parece posible que su aplicación puede tener insospechadas posibilidades, decimos esto a propósito que ella compromete a la idea de vivencia en estado presente (Bergson), por una parte, y a la idea de sentido prospectivo que conllevan los proyectos de acción, por otra parte. Para intentar demostrar esta afirmación nos proponemos aplicar la noción de vivido-proyectado en el ámbito de las relaciones intersubjetivas que establece un profesor y sus estudiantes en la sala de clases. ${ }^{7}$

Los diversos procesos de conocer, de construir conocimiento y de formación en los cuales participan los estudiantes, constituye un ejemplo interesante de revisar, especialmente en relación a los fenómenos intersubjetivos que surgen al momento que un profesor se relaciona con un discípulo o cuando éste se relaciona con sus compañeros de clase.

Los actores que participan en los procesos de aprendizaje constituyen agentes principales en los procesos cognoscitivos que ocurren en el aula; por otra parte, las diversas estrategias metodológicas usadas para lograr algunos propósitos, y que el maestro formula en el contexto de los Planes y Programas de Estudio, a nuestro parecer, desempeñan un rol auxiliar en este tipo de procesos. A lo más, las estrategias, constituyen recursos orientadores de los procesos cognoscitivos que observamos en ese contexto. Decimos esto, ya que si bien es posible utilizar algunos medios auxiliares, audiovisuales por ejemplo, o de participar en una clase de carácter expositiva, no es menos cierto que en ambos casos los logros no necesariamente serán los esperados. Más bien, pareciera que para que el conocimiento sea congruente con los resultados esperados es indispensable que la conciencia, del estudiante y del

\footnotetext{
${ }^{5} \mathrm{Si}$ "fantaseamos", podemos decir que la aplicación de los proyectos futuros son insospechados, en diversos campos donde se observan relaciones intersubjetivas, como es el caso de la sala de clases; las relaciones políticas; las relaciones que ocurren entre un médico y su paciente; las relaciones de negociación en una empresa; las relaciones comerciales, etc. En esta oportunidad sólo aludiremos a las relaciones intersubjetivas que ocurren en la sala de clases: tanto entre el profesor y los estudiantes, como entre los propios estudiantes.

${ }^{6}$ Aquí, el ejercicio y práctica de la tolerancia tiene proyecciones insospechadas. Pero dejemos pendiente, para otras investigaciones, los alcances de la aplicación de la idea de "vivido proyectado" e intentemos comentar algunas observaciones que percibimos en la relación profesor-estudiante.

${ }^{7}$ No obstante, también nos parece posible que este ejercicio se puede realizar en el contexto del conocimiento que construyen los estudiantes, situación que no abordaremos en esta ocasión.
} 


\section{Rubén Leal}

maestro, deben tener la intención de conocer, es decir, él o los actores necesariamente deben tener como intención un propósito cognoscitivo. No es posible obligar a un hijo que mediante el estudio de una materia logre el conocimiento de ella, ya que podrá simular que lo hace cada vez que oye a su padre que se acerca al lugar donde se encuentra. El padre, por su parte, podrá aceptar que su hijo está estudiando. Sin embargo, esta simulación le permitirá al joven lograr el permiso que desea, pero, una vez recibidos los resultados de su examen, encontramos que ha sido reprobado. ¿Cómo se explica el padre que su hijo reprobó su examen, no obstante haber sido testigo del tiempo que el joven dedicó a su preparación?

En una situación como la señalada, es claro que el estudiante no aprendió; es decir, no logró conocer. Una de las razones que se pueden aducir es que el fracaso ocurrió debido a que el hijo nunca estudió y tan sólo simulaba hacerlo cada vez que su padre se aproximaba al lugar de estudio. En este caso, estamos ante un fenómeno donde el actor realiza una representación teatral, pero, de ninguna manera, su proyecto de acción ha comprometido a su conciencia en la formulación de su proyecto, es decir, que el hijo considere el resultado esperado por el padre. En esta situación, por ejemplo, más bien pareciera que la conducta del actor se encuentra dirigida a recordar los momentos de descanso vividos durante las vacaciones en compañía de sus amigos, pero la conciencia no tiene el propósito de prepararse y de conocer aquello que fue exigido en el examen y que tuviera como corolario lo esperado por el padre.

Para que el resultado de un trabajo, de preparación de un examen por ejemplo, sea de aprobación y no una mera simulación, es menester que la conciencia del hijo haya seleccionado ese objetivo y, a su vez, que tenga la intención de conocer. En este tipo de situaciones, el propio estudiante, junto con imprimirle una dirección a su conciencia, también habrá establecido un proyecto que tiene como resultado vivencias cognoscitivas, es decir, los contenidos del proyecto serán parte de su conciencia y, por otra parte, ello traerá consigo la alta probabilidad de que, en esas circunstancias, el estudiante apruebe su examen y deje satisfecho a su padre. Sólo aquí estaremos ante una situación donde el estudiante construyó conocimiento.

Por su parte, si observamos que ambas conciencias — del profesor y del estudiante - se encuentran dispuestas a compartir las vivencias que cada uno ha tenido en el transcurso de su vida estudiantil y profesional, respectivamente, entonces el maestro y el discípulo habrán establecido relaciones intersubjetivas. Más aún, pareciera que no es posible lograr los propósitos establecidos en la planificación y en la organización de una clase si, junto con ello, no se observa la disposición e incluso el compromiso - consigo mismo- de establecer un proyecto de acción que comprometa los aspectos que conforman el proyecto personal de cada actor (del maestro y del discípulo). Parece posible, y legítimo, que el modo y las acciones que conforman el proyecto - cognoscitivo - de ambos puedan ser diferentes; sin embargo, también parece posible de consensuar, al menos, algunos aspectos relevantes que forman parte de los proyectos personales de cada actor. Si sucede lo 
último, podemos pensar que nos encontramos ante un proyecto de acción que es común y en ese proyecto, mediante un esfuerzo consciente e intencionado de ambos, se logrará integrar las aspiraciones futuras que ambos actores tenían consideradas de manera particular. En este caso, y por otra parte, maestro y discípulo han decidido postergar algunas consideraciones o aspiraciones - personales - con el solo propósito de establecer una acción común.

Con un criterio como el señalado, de haber logrado establecer un proyecto común, es posible de distinguir la existencia consciente, intencionada e integrada, de las vivencias personales de ambos actores. Claramente, en este caso, las vivencias del estudiante han constituido situaciones distintas de aquellas vividas por el maestro; es decir, por una parte, nos encontramos con vivencias que no son similares - para el maestro y para el discípulo- y, por otra parte, el uso que de ellas pueda realizar uno u otro actor también podrá ser diferente. La edad cronológica, las experiencias educativas y profesionales, incidirán de manera sustancial en el uso de las vivencias que realice uno $u$ otro actor.

Sin embargo, y tal como lo habíamos señalado, tanto el maestro como el discípulo pueden decidir establecer un tipo de relación muy especial, intersubjetiva; es decir, ambos y gracias a esta opción, podrán formular un proyecto que tiene como propósito lograr el conocimiento, ya sea debido a que uno optó por asistir a clases o debido a que el otro se ha dedicado a preparar actividades, entre las cuales ha seleccionado recursos bibliográficos que ayudan a que maestro y discípulos tengan la posibilidad de vivenciar una serie de experiencias que, a su vez, facilitarán logros cognoscitivos. De suceder lo último, pareciera que el estudiante puede recurrir a sus vivencias para formular nuevos proyectos $\mathrm{y}$, también, asumir las diversas circunstancias que vivirá en el futuro con un criterio que facilitará sus propósitos, con todo lo que ello significa para su vida personal. Aquí nos encontramos con lo que hemos asumido como su acervo de conocimiento a mano. ${ }^{8}$ Quizás, este sea el momento de preguntarnos acerca de ¿Cómo es posible que el profesor sepa que el resultado de las relaciones que establece en la sala de clases, con sus estudiantes, tenga como corolario el conocimiento como parte de la formación de sus discípulos?

Una manera de intentar despejar esta duda es interrogándonos previamente acerca de ¿Cuáles son las estrategias que debería utilizar el profesor para tener algún grado de certeza - a priori - que su propósito profesional tenga el resultado esperado?

Pareciera que el maestro, primeramente, se ve impelido a conocer los proyectos de acción de sus estudiantes; es decir, en el contexto de la cotidianeidad académica el profesor deberá reconocer que el otro es un actor, distinto de él; que tiene una biografía diferente a la suya y que sus raíces se encuentran más allá del

\footnotetext{
${ }^{8}$ Recordemos que las vivencias se encuentran en estado presente en la propia conciencia del estudiante $\mathrm{y}$, desde otro punto de vista, ellas mismas constituirán importantes insumos que el actor usará en el transcurso de su vida inmediata y mediata.
} 


\section{Rubén Leal}

ámbito puramente escolar. El estudiante ha tenido experiencias y vivencias en su vida diaria y escolar que también son distintas de las que ha tenido y tiene su maestro. Así, aceptar que el estudiante vive en un ámbito cotidiano distinto del escolar y que al mismo tiempo necesita de su maestro —en el contexto académico, profesional y formativo, en general - para lograr sus objetivos educativos requiere, adicionalmente, asumir que el Profesor se encuentra con el problema de alcanzar sus propósitos profesionales. Junto con reconocer la subjetividad de su discípulo - y la suya- el maestro tendrá que interesarse por las aspiraciones que tiene; reconocer que el otro posee características similares a las suyas, pues, en la medida en que percibe al estudiante como a un otro con diferencias y similitudes a las suyas, que son seres conscientes, entonces es posible que establezca relaciones intersubjetivas y formule proyectos comunes con su discípulo. Aquí observamos una dificultad adicional, cual es, que el estudiante también asuma al profesor como un actor similar a sí y del cual necesita si pretende alcanzar sus propósitos escolares y formativos. Esto último, considerando las ventajas cronológicas, vivenciales y profesionales del profesor que, a su vez, deberán ser parte de las estrategias que utilizará en su desempeño diario.

La noción de vivido-proyectado, entonces, puede ayudar al profesor a conocer a su discípulo, especialmente en el estudio y en la elaboración de su biografía, toda vez que el conocimiento de las conductas que el estudiante ha tenido en su vida y el modo como ha ido asumiendo las circunstancias que han sido señeras y relevantes en su existencia, permitirán al maestro realizar una interpretación adecuada de los proyectos que el estudiante se propone. Un conocimiento de la biografía del otro y un reconocimiento de él como un otro que es similar, pueden ayudar significativamente tanto en la interpretación como en el conocimiento del yo de la conciencia de cada actor social. Desde un estudio biográfico (Pujadas, 2002:41-57), por ejemplo, nos parece posible acceder al conocimiento del proyecto de acción del otro y desde el conocimiento del otro parece posible establecer proyectos comunes entre dos o más actores, en este caso entre maestro y discípulo.

\section{LAS VIVENCIAS DEL ACTOR EN EL PROCESO DE PROYECTAR LAS ACCIONES SOCIALES}

Cuando afirmamos que las acciones tienen como contenido a los actos futuros que realizará un actor pareciera que surgen dos aspectos de un mismo fenómeno. Por una parte, aludimos al rol que desempeña la conciencia, que parece distinto a cuando ella se vincula con el acto que ejecuta un actor y, por otra parte, nos referimos al contenido de las acciones, que también parecieran ser diferentes del contenido de los actos. Esta distinción nos surge a propósito de que las acciones tienen su propio sello, el que pareciera que depende de la función que cumple la conciencia y del contenido que las propias acciones tienen. En relación a lo segundo, si tenemos a la vista el hecho de que el contenido de una acción corresponde a las vivencias - en estado presente - entonces no tenemos más alternativa que aceptar la distinción aludida, 


\section{La idea de "vivido-proyectado"}

claro, asumiendo que el estado presente de una vivencia, en la conciencia del Sujeto, nos lleva en algunas ocasiones a confundir a la acción con el acto.

Sin embargo, dejemos establecido que los actos tienen como insumo y privilegian a los proyectos en su totalidad, o a parte de ellos, cada vez que la conciencia procede a seleccionarlos. Para acotar este punto, traigamos a la discusión los comentarios que Alfred Schütz realiza cuando se refiere al fenómeno de las acciones (1993:174-189).

a) La primera distinción se refiere a las acciones de las cuales no se ha realizado reflexión alguna, es decir, aquí Schütz alude a las acciones que se caracterizan por su espontaneidad y en este grupo incluye a aquellas (acciones) que no han sido planteadas por un actor, al menos antes de ser ejecutadas.

b) En el segundo grupo, Schütz considera aquellas acciones de las cuales sí se ha reflexionado, de manera que en este tipo de casos la conciencia opera de un modo distinto del anterior.

Recordemos que la reflexión proyectiva implica una acción futura que es asumida como si hubiese ocurrido; es decir, en los procesos de proyectar, la acción es vista como un fenómeno que ha concluido plenamente y en el desarrollo de ese proceso se puede recurrir, básicamente, a la técnica de fantasear, lo que permitirá contar con los recursos suficientes para realizar un acto reflexivo-proyectivo. Así, tenemos que la acción-proyectiva aparece como si careciera de contenido, en el sentido que esa acción viene a ser, con su contenido, la que puede ser adscrita a la conciencia intencional que organiza las vivencias. ${ }^{9}$ De aquí es que podemos decir que la acción proyectiva integra-el-pasado-y-el-tiempo-futuro de un modo intencional y selectivo, especialmente, debido a que la conciencia cuando procede a proyectar opera de manera prospectiva. Sin embargo, y en forma implícita al menos, se debe reconocer que la conciencia también contempla en su acto de fantasear algunos aspectos vivenciales que han ocurrido en tiempo pasado.

Pero volvamos a las distinciones señaladas precedentemente, entre acción y acto, y tengamos presente las consideraciones recién aludidas, pues ambos aspectos nos permiten inferir que todas las acciones no tienen un sentido prospectivo, ya que algunas son espontáneas y otras son planeadas; por lo cual, surge una nueva distinción en que a unos proyectos los podríamos llamar acciones fantasiosas y, otros, acciones reflexivas, ya que en el segundo grupo se ha procedido a reflexionar antes de su construcción. En este segundo grupo de acciones, también se debe reconocer que ellas han incorporado aspectos fantasiosos que simulan su ejecución; no obstante, sea de uno o de otro tipo, habría que reconocer que la acción fantasiosa no corresponde a una acción propiamente tal, pues ella alude a un mero recurso para formular un proyecto que, a su vez, no es dependiente de la acción propiamente tal.

\footnotetext{
${ }^{9}$ Ellas ocurrieron en tiempo pasado y para los efectos del acto de fantasear, al menos parte de las vivencias fueron seleccionadas en virtud de su pertinencia con los propósitos de la acción que se llevará a cabo.
} 


\section{Rubén Leal}

En cuanto a las fantasías, aceptemos que ellas son parte de las acciones a las cuales nos hemos referido hasta aquí; también, asumimos que contienen aproximaciones intuitivas con un importante grado de vaguedad, como es el caso de las acciones que han sido ejecutadas. En este punto, y en rigor, pareciera que sólo podemos hablar de proyectar cuando nos referimos a una acción, ya que el acto constituye una especie de procedimiento mediante el cual se lleva a cabo o se ejecuta una acción determinada. Así, tenemos que la acción se convierte en acto al momento que se ejecuta en la vida cotidiana.

Entonces, el significado que contiene una acción viene a ser el acto que se lleva a cabo, de manera que entre la acción y el acto se observa un estrecho vínculo, no obstante ambos corresponden a fenómenos diferentes. Esta vinculación, a nuestro parecer, constituye un fenómeno epistemológicamente esencial, toda vez que alude a una especie de unidad subjetiva - en el lenguaje y categorías schutzeanas - y que, en este caso, el actor procede a proyectar o a formular la acción y, a su vez, él mismo procede a ejecutar el acto. De manera que el actor inicia y cierra esta especie de círculo o de sistema: una acción al ser pensada pasa a transformarse en acto y sólo al actor le es posible conocer el origen, el proceso y el momento en el cual se cierra el sistema que integra, tanto a la acción como al acto. Ahora se nos aclara la relación que establece la conciencia entre el proyecto de acción y el acto ejecutado.

No obstante lo anterior, y en atención a su relevancia, detengámonos un instante para comentar la relación que establece la conciencia con la acción de proyectar. Decíamos que el fundamento que sustenta la relación entre acción y acto es la "unidad subjetiva" que, en el caso de la acción, el actor recurre al acto de fantasear $\mathrm{y}$, conforme a este recurso, procede a formular un proyecto, en tiempo futuro perfecto. Sin embargo, una vez más, se nos presenta un problema relacionado con la acción y el acto: nos referimos al momento cuando se pretende proyectar una acción específica. Volvamos al proyecto para ver si logramos superar este impedimento, al menos, en cuanto a lo que ahora nos interesa.

El proyecto es una especie de configuración compuesta de significado/s y que opera en virtud de su vínculo con la acción; es decir, no hay acción sin proyecto y no es posible el acto sin la acción. Cuando procedemos a proyectar, la conciencia y nuestra capacidad reflexiva realizan un tipo de actividad muy especial cuya característica básica la encontramos en el sentido constituyente, el que permite, a su vez, ejecutar, el acto de proyectar. No obstante, precedentemente también habíamos aludido a esa especie de sistema que conforma la acción y el acto, por lo cual intentemos ahora observar si antes de la acción existen insumos que apoyan su constitución y su respectiva proyección.

Antes de la formulación de un proyecto, señalamos que el actor había articulado las vivencias que sirven de sustrato; también habíamos declarado que ellas no obstante haber sido vividas en circunstancias y momentos diversos son parte del pasado, en un sentido factual. El actor, siempre podrá recurrir a sus vivencias al 
momento de formular proyectos de futuros actos; ello, principalmente debido a que la conciencia, como decíamos, mantiene a las vivencias en estado presente. Sin embargo, aquí podríamos preguntarnos ¿Cómo es posible que el actor mantenga en estado presente situaciones que fueron experimentadas en tiempo pasado? ¿Significa que en la conciencia del actor existe un caos de representaciones? ${ }^{10}$

Una explicación posible sería pensar que la conciencia, al no ser asumida con un criterio topográfico y que se queda con representaciones de algunas experiencias, viene a constituir y a recurrir al "acervo de conocimiento" que, como sabemos, no ocupa un lugar espacial en ella. La conciencia es la capacidad del actor para darse cuenta de la o de las experiencias vividas, de aquí que a ella le es posible iniciar procesos que llevan a que algunas experiencias se constituyan en vivencias, en la conciencia del actor.

Otro modo de responder nuestras preguntas, sería pensar que si bien el actor ha procedido a articular las vivencias y, por otra parte, aceptando que ellas se encuentran en estado presente en su conciencia, entonces ese estado se puede interpretar como una especie de esquema representativo gracias al cual, y con la ayuda de su memoria, el actor procede a reconstruir la o las vivencias que le sirven de insumo en la formulación de un proyecto; es decir, en la conciencia nos podemos encontrar con esquemas que presentan ciertos niveles de complejidad y que constituyen una especie de llave que abre la posibilidad de llegar a las propias vivencias. Por su parte, el nivel de complejidad de los esquemas que representan a las vivencias dependerá del momento en que se vivió la experiencia; dependerá, también, de la importancia que el actor le otorgue a tal suceso y de las opciones que tuvo al momento de seleccionar algunos o todos los aspectos que conformaban la situación vivida. Así, se puede decir que cuando el actor formula un proyecto toma como insumo los acontecimientos que sucedieron en el pasado mensurable y estos actos, al ser parte de su conciencia (por cuanto la conciencia es siempre conciencia de algo, como señala Husserl) pasan también a ser parte del presente, en el contexto de un tiempo que no es posible de medir (Bergson, 1993). En general, pareciera que ahora nos encontramos ante una o unas motivaciones que afectan al actor, desde un pasado, a un proyecto que tiene un sentido prospectivo (de aquí la teoría de los motivos, especialmente de los motivos para).

Desde otro punto de vista, es posible pensar que en la formulación de proyectos con sentido de futuro, es decir, en la evidencia del acto de proyectar, observamos dos tipos de tiempo: uno, relacionado con la idea bergsoniana (de tiempo interno y tiempo externo) y un segundo tipo de tiempo vinculado a la expresión de una acción pasada (que Schütz llama pluscuamperfecto) que integra o que tiene un sentido de futuro. Parafraseando a Schütz, podemos decir que el actor sólo conoce el motivo porque después de haber completado la acción proyectada, y que el acervo de

\footnotetext{
${ }^{10}$ Cada representación alude a una experiencia vivida en el pasado y que se ha transformado en vivencia gracias al acto consciente que se ha tenido de ella.
} 


\section{Rubén Leal}

conocimiento opera de manera automática (metafóricamente hablando). De modo similar, el acto intencional de la conciencia — que selecciona parte o alguna/s vivencia/s - opera de un modo inconsciente cuando se procede a proyectar, es decir, en el último instante del proceso de proyectar el actor sólo tiene la posibilidad de recurrir al expediente de tematizar el proyecto que él mismo formula.

Por lo expresado, podemos sostener que las acciones que se realizarán tienen una lógica motivacional, en el sentido que en ellas encontramos una acción motivante, un proyecto de acción y un acto motivado, entendiendo este último como la consumación de todo aquello que observamos en esta lógica. La acción que motiva viene a ser la función intencional que desempeña la conciencia cuando ella, ante sí misma, se propone seleccionar y formular un propósito determinado, que tiene en cuenta los deseos y/o necesidades del actor. El proyecto motivante, por su parte, constituye el factor integrador de los deseos y/o de las necesidades para realizar un acto, donde el primero usará todos aquellos recursos vivenciales que sean necesarios para alcanzar este propósito. Aquí, la conciencia recurrirá al expediente de fantasear como una técnica necesaria para anticiparse a lo que sucederá. La acción de fantasear, por su parte, no debemos entenderla como una causa del acto, sino más bien como un proceso donde el acervo de conocimiento es un recurso que sirve para contextualizar y para encontrar los insumos que permiten establecer un proyecto de acción. El acto motivado será la ejecución del proyecto, en tanto éste viene a ser la fantasía de ese acto ejecutado.

\section{CONCLUSIONES}

Una de las cuestiones de las cuales nos hemos ocupado, aquí, es tratar de comprender por qué Schütz no avanza en el estudio de la teoría de los motivos; especialmente intentamos interpretar el sentido y los alcances epistemológicos que tiene el carácter prospectivo de sus reflexiones sobre los motivos para. Ahora sabemos que este autor no trasciende en su crítica lo planteado por Weber, pero al iniciar esta investigación nuestro interés principal era encontrar algunos aspectos que nos permitieran referirnos a la noción de vivido-proyectado, pues, con esta idea pensábamos que era posible encontrar una herramienta para interpretar los aspectos que forman parte de los proyectos de acción que formulan los actores sociales. También nos proponíamos ver el modo como ellos comparten la vida cotidiana, formulan proyectos en conjunto y construyen relaciones sociales desde la tolerancia.

El proyecto de acción, varias veces señalado aquí y en otros trabajos, es una especie de propuesta teórica que realizan los actores para ejecutar sus actos en la vida cotidiana, especialmente en los procesos que viven con otros actores. Estas relaciones tienen un carácter subjetivo. Por eso, compartimos y nos apoyamos en la propuesta de intersubjetividad que propone Schütz de las relaciones sociales intersubjetivas. En este sentido, también hemos asumido que, si bien la conciencia del actor cumple un rol esencial en la organización y en la mantención de las vivencias en estado presente, no es menos cierto que en las relaciones intersubjetivas ella cumple un rol sustancial en la formulación de los proyectos que le son comunes. Lo primero, nos permite 
explicar el sentido como hemos asumido la idea de acervo de conocimiento y, lo segundo, nos abre la posibilidad de interpretar ese tipo de relaciones con una visión proyectiva, es decir, la conciencia nos ayuda a comprender los procesos de construcción de relaciones sociales, de un modo participativo. Esto último lo hemos abordado, en este trabajo, especialmente en el acápite sobre las aplicaciones de la idea de vivido-proyectado y deseamos continuar nuestras investigaciones en las posibles aplicaciones que esta noción puede tener.

Ahora sabemos que, gracias a la conciencia y mediante la acción, el actor procede a otorgarle sentido a cada acto que realiza, pues la conciencia es usada con el propósito de interpretar subjetivamente las vivencias que ha tenido cada actor y, a la vez, definir el contexto donde realizará el acto. En este aspecto, debemos estar alerta ya que las acciones y los proyectos que las contienen no son hechos aislados que ocurren en la vida de los sujetos; por el contrario, las acciones se perciben de manera concatenada en el desarrollo de la vida cotidiana de ellos y de nosotros. Con un sentido epistémico, nos parece que las acciones adquieren mayor relevancia en el contexto de la teoría de los motivos, especialmente en el ámbito de los estudios acerca de los motivos para, realizados por Schütz. Por su parte, si bien Schütz no se ocupa de la naturaleza de las razones que motivan a una acción y a un acto determinado, lo que parece más relevante es señalar que con las interpretaciones realizadas sobre este punto es posible continuar el trabajo iniciado por Weber y Schütz. Particularmente, nos referimos a la interpretación de las acciones y al estudio de los actos futuros que realizarán los actores. Seguir la línea de trabajo inaugurada por estos autores, desde la noción de vivido-proyectado, por tanto, trascender el sentido topográfico e interno que la tradición positivista le otorga a las vivencias (en tanto es imposible acceder a ellas por parte de otro sujeto) nos parece una tarea importante. Consideramos que investigar el proyecto de acción desde esta herramienta nos abre caminos interesantes desde una perspectiva epistémica y también ético-política y educativa.

\author{
Universidad de La Frontera* \\ Departamento de Ciencias Sociales \\ Casilla 54-D, Тетисо (Chile) \\ rleal@ufro.cl
}


Rubén Leal

\section{BIBLIOGRAFÍA}

Bech, Joseph María. De Husserl a Heidegger. Transformación del pensamiento fenomenológico. Barcelona: Universidad de Barcelona, 2001.

Bergson, Henri. Obras escogidas. (Traduc., y Prólogo de José Antonio Míguez). Madrid: Aguilar, 1993.

Habermas, Júrgen. Teoría de la acción comunicativa. T. I - II. Madrid: Taurus, 1987.

Hegel, G.W.F. Ciencia de la Lógica. (Traduc., y Prólogo de Rodolfo Mondolfo. Edic. 6ta.). Buenos Aires: Ediciones Soler, Librería Hachette, S. A. R. I., 1968.

Husserl, Edmundo. Investigaciones lógicas (II). (Traduc. Manuel García Morente y José Gaos). Barcelona: Atalaya, 1995.

------ Ideas relativas a una fenomenología pura y una filosofía fenomenológica. (Traduc. José Gaos). Segunda reimp. México: F.C.E., 1992.

Leal, Rubén; Herrera, Berta. "La constitución de significado en el ámbito de las relaciones intersubjetivas: El acto personal y la acción social", en Alpha $\mathrm{N}^{\circ} 28$. 2009:135-151.

Leal, Rubén. "La sociología interpretativa de Alfred Schütz. Reflexiones en torno a un planteamiento epistemológico cualitativo", en Alpha $\mathrm{N}^{\mathrm{0}}$ 23. 2006:201-212.

"Análisis del desarrollo social intersubjetivo desde las nociones de mundo de la vida y mundo de la ciencia, propuestos por Alfred Schütz", en Alpha No 19. 2003:263-275.

------ "El problema de la intersubjetividad. Aproximaciones a la propuesta epistemológica de A. Schütz", en Alpha No 17. 2001:215-229.

León, Emma; Zemelman (Coordinadores). Subjetividad: umbrales del pensamiento social. Barcelona: Anthropos, 1997.

Pujadas, Juan José. El método biográfico: el uso de las historias de vida en ciencias sociales. Madrid: Centro de Investigaciones Sociológicas. 2002.

Schütz, Alfred. El problema de la realidad social. Mauricio Natanson compilador. Buenos. Aires: Amorrortu, 1995.

------ La construcción significativa del mundo social. Introducción a la sociología comprensiva. La edición anterior fue publicada como Fenomenología del mundo social. Traduc. Eduardo Prieto. Pról. Joan-Carles Mélich. Primera reimp. Barcelona Paidós. 1993.

------ Estudios sobre teoría social. (Traduc. Ricardo Miliandi. Compilador Arvid Brodersen). Buenos. Aires: Amorrortu. 1974.

Weber, Max. Economía y sociedad. Esbozo de sociología comprensiva. (Traduc. José Medina Echevarría et al). Décima reimp. México: F.C.E., T.I., 1969. 